\title{
Circulating levels of calprotectin, a signature of neutrophil activation in prediction of severe respiratory failure in COVID-19 patients: a multicenter, prospective study (CalCov study)
}

\author{
Luis García de Guadiana-Romualdo ${ }^{1}$ (1) - Carlos Rodríguez Rojas² ${ }^{2}$ Daniel Morell-García ${ }^{3,4}$ - David Andaluz-Ojeda ${ }^{5}$ \\ María Dolores Rodríguez Mulero ${ }^{6}$. Enrique Rodríguez-Borja ${ }^{2} \cdot$ Antonieta Ballesteros-Vizoso $^{3} \cdot$ María Dolores Calvo $^{7}$. \\ Lourdes Albert-Botella ${ }^{8}$. Adela Pozo Giráldez $z^{2}$ Isabel Llompart-Alabern ${ }^{3}$. Cristina Bolado Jiménez ${ }^{9}$. \\ Marta Hernández Olivo ${ }^{10}$. Lucía Fernández Presa ${ }^{11}$. Juan Ortega-Pérez ${ }^{4,12}$. María José Fora Romero ${ }^{9}$. \\ Valerio Campos-Rodríguez ${ }^{13}$. Andrea Ballester Férriz ${ }^{11}$. Alexandra M. Guiu-Martí ${ }^{12}$. Leyre Pinilla Arribas ${ }^{9}$. \\ María Galindo Martínez ${ }^{6}$. Edwin R. Puerto-Lara ${ }^{12}$ - Ana Belen López Tarazaga ${ }^{9}$ María Salomé Ros Braquehais ${ }^{10}$. \\ Leonor Nogales Martín ${ }^{5}$. Celia Juez Santamaría ${ }^{14}$. Wysalli Trapiello Fernández ${ }^{9}$ Virginia Espinilla Fernández ${ }^{15}$. \\ Aleksandra Havelka $^{16,17}$. María Dolores Albaladejo-Otón ${ }^{1}$
}

Received: 17 May 2021 / Revised: 21 October 2021 / Accepted: 23 October 2021 / Published online: 30 October 2021

(c) The Author(s), under exclusive licence to Springer Nature Switzerland AG 2021

\begin{abstract}
Objective Severe COVID-19 is characterized by a dysregulated immune response in which neutrophils play a critical role. Calprotectin reflects neutrophil activation and is involved in the self-amplifying thrombo-inflammatory storm in severe COVID-19. We aimed to evaluate the role of calprotectin in early prediction of severity in COVID-19 patients.

Methods This was a multicenter prospective observational study enrolling consecutive adult COVID-19 patients. On arrival to emergency department, blood samples were collected for laboratory tests, including serum calprotectin. The primary outcome was severe respiratory failure requiring invasive mechanical ventilation and the secondary outcome was need for Intensive Care Unit (ICU) admission.

Results Study population included 395 patients, $57(14.4 \%)$ required invasive mechanical ventilation and $100(25.3 \%)$ were admitted to ICU. Median serum calprotectin levels were significantly higher in intubated ( $3.73 \mathrm{mg} / \mathrm{L}$ vs. $2.63 \mathrm{mg} / \mathrm{L} ; p<0.001)$ and ICU patients $(3.48 \mathrm{mg} / \mathrm{L}$ vs. $2.60 \mathrm{mg} / \mathrm{L} ; p=0.001)$. Calprotectin showed a significant accuracy to predict the need for invasive mechanical ventilation (ROC AUC 0.723) and ICU admission (ROC AUC 0.650). In multivariate analysis, serum calprotectin was an independent predictor of invasive mechanical ventilation (OR 1.161) and ICU admission (OR 1.068). Conclusion Serum calprotectin can be used as an early predictor of severity in COVID-19 patients.
\end{abstract}

Keywords Calprotectin · COVID-19 · Neutrophil activation · Invasive mechanical ventilation · ICU admission

\section{Introduction}

In December 2019, an outbreak of pneumonia due to a novel coronavirus, severe acute respiratory syndrome coronavirus 2 (SARS-CoV-2), occurred in Wuhan (Hubei Province, China). The disease caused by this virus was named

Responsible Editor: Anatoliy Kubyshkin.

Luis García de Guadiana-Romualdo

guadianarom@yahoo.es

Extended author information available on the last page of the article
Coronavirus disease 19 (COVID-19) and has resulted in a pandemic affecting millions of people globally [1].

The clinical presentation of COVID-19 varies from asymptomatic or mild upper respiratory tract symptoms to severe viral pneumonia with acute respiratory distress syndrome (ARDS) and need for mechanical ventilation. Data from a Spanish multicenter study including 4035 hospitalized patients with confirmed COVID-19 showed the development of ARDS in 31.5\% and need for mechanical ventilation in $15.5 \%$ of patients [2]. An early identification upon hospital admission of COVID-19 patients at high risk of progression to the most severe forms of the disease is key to 
define an optimal strategy for the management of COVID-19 patients and efficient use of hospital resources [3].

The pathophysiology of severe COVID-19 is characterized by neutrophil activation [4] and hyperinflammatory host response, which potentially lead to endotheliitis [5], severe respiratory failure and mortality [6]. Hence, an aberrant neutrophil response in COVID-19 is likely due to uncontained viral replication, tissue hypoxia and exacerbated inflammation [7]. One of the main effector mechanisms of neutrophils in inflammatory and infectious diseases are neutrophilderived extracellular traps (NETs) [8], which are thought to be part of an innate immune mechanism for pathogen clearance; however, there are increasing concerns about the potential of NETs to initiate and propagate inflammatory damages in host tissues [9], including lungs, resulting in reduced oxygenation and ARDS in COVID-19 patients [9]. Veras et al. [10] have demonstrated that in patients with severe COVID-19, circulating and lung-infiltrating neutrophils release higher levels of NETs and this release is stimulated directly by SARS-CoV-2 virus. The authors indicate that the potentially deleterious role of NETs for lung epithelial cells might explain partially the pathophysiology of severe COVID-19 [10].

Calprotectin, as a signature of neutrophil activation, has emerged as a promising biomarker for risk stratification in COVID-19 patients during the first wave of pandemic [11, 12]. Calprotectin is a heterodimeric protein composed of S100A8 and S100A9 proteins (also called myeloid-related proteins (MRP) 8/14), belonging to the S100 family of calcium-binding proteins. Increased calprotectin levels have been reported in inflammatory $[13,14]$ and infectious diseases [15-17]. It is particularly abundant in the cytoplasm of neutrophils and may also be detected at low levels in other cells, such as monocytes and macrophages [18]. In COVID-19 patients, Shi et al. [19] have described markedly increased serum calprotectin levels in patients with severe form of the disease indicating the role of neutrophils as potential players in COVID-19 severity.

In addition to being a neutrophil-related inflammatory marker, calprotectin may also have a direct role in the self-amplifying thrombo-inflammatory storm observed in patients with severe COVID-19. Silvin et al. [20] have reported that excess production of damage-associated molecular patterns (DAMPs) drives this association and showed that elevated calprotectin levels are linked to immature neutrophils and nonclassical monocytes. Therefore, calprotectin has been suggested to be a key mediator in the hyperinflammatory host response and expansion of inflammatory monocytes, neutrophils and platelets contributing to the characteristic coagulopathy in severe COVID-19 [21].

Cherubini et al. [22] have recently reported higher plasma calprotectin levels in patients with confirmed infection by SARS-CoV-2 in comparison to suspected patients with negative RT-PCR. The role of calprotectin in evaluation of disease severity has been confirmed in several studies [19, 23-26], although these findings should be assessed in larger prospective cohorts [12]. Since severe cases of COVID-19 are regularly complicated by respiratory failure, in this multicenter study, we aimed to evaluate the role of circulating serum calprotectin, measured at hospital admission, as an early predictor of severe respiratory failure and ICU admission in hospitalized patients infected by SARS-CoV-2.

\section{Materials and methods}

\section{Study design and patient selection}

This was a multicenter prospective observational study enrolling consecutive adult ( $\geq 14$ years) COVID-19 patients admitted to Son Espases University Hospital (Palma de Mallorca, Spain), Clinic University Hospital (Valencia, Spain), Clinic University Hospital (Valladolid, Spain) and Santa Lucía University Hospital (Cartagena, Spain) during the second wave in Spain (September to November 2020). Infection by SARS-CoV-2 was diagnosed by a positive result of real-time reverse transcriptase-polymerase chain reaction testing of a nasopharyngeal or lower respiratory tract specimen, according to WHO Guidance [27]. Exclusion criteria were: (a) patients $<14$ years; (b) pregnant women; (c) patients transferred from or to other hospital; (d) length of hospital stay $<24 \mathrm{~h}$; (e) limitation of therapeutic effort or life-sustaining treatment; and (f) lack of samples for the measurement of biomarkers. Only a first episode of admission to hospital was considered for inclusion in this study.

The study protocol was approved by the local Ethics Committee of participating hospitals (E.0.2020-38 LAB2COVID19) and was performed under a waiver of informed consent and in accordance with the Declaration of Helsinki ethical guidelines.

\section{Study endpoints}

The primary outcome was severe respiratory failure requiring invasive mechanical ventilation. Because there is no single number or algorithm that determines the requirement for invasive mechanical ventilation, the determination of need for endotracheal intubation for COVID-19-related respiratory failure was made by clinicians considering a variety of factors such as signs of unsustainable work of breathing, refractory hypoxemia, hypercapnia or respiratory acidosis and encephalopathy or inadequate airway protection [30]. Secondary endpoint was need for admission to Intensive Care Unit (ICU) during the hospital stay. 


\section{Data collection}

For eligible patients, epidemiological, demographic, clinical, laboratory, home and in-hospital treatment and outcome data, including ventilation status and ICU admission, were extracted from electronic medical records and laboratory information systems.

\section{Blood sampling and laboratory analysis}

In all patients, blood samples were drawn upon arrival to the Emergency Department and then centrifuged $(3000 * \mathrm{~g}$ for $5 \mathrm{~min}$ for non-anticoagulated blood to obtain serum and $1500 * \mathrm{~g}$ for $15 \mathrm{~min}$ for blood samples containing trisodium citrate as anticoagulant to obtain plasma for coagulation testing) and analysed within $1 \mathrm{~h}$ from collection. According to recommendations from Spanish Health Ministry [28], the following laboratory tests were measured: creatinine, by a colorimetric assay (Jaffe reaction), C-reactive protein (CRP), by an immunoturbidimetric assay, and lactate dehydrogenase (LDH), by an ultraviolet assay, on Architect ci16200 (Abbott Diagnostics, US) and Cobas c502/702 (Roche Diagnostics, Germany) platforms, D-dimer, by immunoturbidimetry on ACL-TOP Family (Instrumentation Laboratory, US) and Stago STA-R (Stago Diagnostica, Belgium) analyzers, and cell blood count (CBC) by flow cytometry on Sysmex XN (Sysmex, Japan) and Cell-Dyn Saphire (Abbott Diagnostics, US) analyzers. For calprotectin and ferritin, leftover serum was immediately frozen and stored at $-80{ }^{\circ} \mathrm{C}$ until analysis. Serum ferritin levels were measured by a chemiluminescence immunoassay and turbidimetry on Architect ci16200 (Abbott Diagnostics, US) and Cobas c502/702 (Roche Diagnostics, Germany) platforms, respectively. Serum calprotectin levels were measured by a particle enhanced turbidimetric immunoassay (PETIA) (Gentian AS, Norway) using a Cobas c702 instrument (Roche Diagnostics, Mannheim, Germany). According to manufacturer's data, limit of quantification is $0.30 \mathrm{mg} / \mathrm{L}$ and the measurement range is $0.43-20.66 \mathrm{mg} / \mathrm{L}$, with automatic dilution of samples with higher concentrations. Upper normal reference limit (UNRL), obtained from analysis of serum samples from 100 healthy individuals using a Cobas c501 analyzer (Roche Diagnostics, Mannheim, Germany), is $1.63 \mathrm{mg} / \mathrm{L}$ (data not published). According to Nilsen et al. [29], the imprecision was lower than $1.5 \%$ for samples ranging from $1.17 \mathrm{mg} / \mathrm{L}$ up to $22.14 \mathrm{mg} / \mathrm{L}$.

\section{Statistical analysis}

The normality of continuous variables was tested by Kolmogorov-Smirnov and they are reported as median (interquartile range [IQR]). Categorical variables are presented as frequency and percentage in each category. Mann-Whitney
$U$ and chi-squared tests were used to compare continuous and categorical data between groups. Kruskal-Wallis and chi-squared tests were used to analyse the association of calprotectin, stratified according to quartiles, with invasive mechanical ventilation and other population characteristics. Correlation between calprotectin and other markers for thrombo-inflammation was evaluated by Pearson correlation coefficient (r).

For association among potential predictors and outcomes, a binary logistic regression was performed and odd ratios (OR) calculated. For multivariable regression, analysis was fitted using two criteria for selection of predictors: clinical relevance and statistical significance in univariate analysis $(p<0.05)$ after testing for collinearity using the variance inflation factor (VIF).

Discriminatory ability for invasive mechanical ventilation and ICU admission was evaluated by calculating the area under the receiver operating characteristic curve (ROC AUC). Optimal cut-offs were defined as the value maximizing the Youden index; furthermore, we determined a second optimal cut-off point aimed to rule out need for mechanical ventilation with a negative predictive value (NPV) of $90 \%$ or greater and sensitivity of at least $85 \%$. In a further step, the impact of calprotectin on the primary endpoint was assessed using Kaplan-Meier curves and the Mantel-Haenszel logrank test. Time was censored at 94 days following admission to the Emergency Department, corresponding to the highest in-hospital length of stay. We performed analyses using the software packages SPSS 21.0 (SPSS Inc., IL, USA) and MedCalc 15.0 (MedCalc Software, Ostend, Belgium). In all tests, a two-sided $p$ value of $<0.05$ was considered significant.

\section{Results}

\section{Baseline characteristics}

Baseline characteristics in the overall cohort, including a total of 395 patients, and stratified data according to need for invasive mechanical ventilation and ICU admission are listed in Supplementary Table 1. Median age in the cohort was 62 years [Interquartile range (IQR) 49-77; range $17-99$ years $)$ and $63.3 \%(n=250)$ of patients were male. The most prevalent comorbidities were hypertension, dyslipidemia and obesity. In the overall cohort, $34.2 \%$ of patients were on ACEI/ARBs, $31.1 \%$ on statins and $20.0 \%$ on antidiabetics.

Concerning outcomes, 57 patients (14.4\%) required invasive mechanical ventilation and 100 patients $(25.3 \%)$ were admitted to the ICU. The median length of hospital stay was 9 days (IQR 6-17; range 1-94). As expected, in-hospital length of stay [8 days (IQR 5-13) vs. 32 days (IQR 21-42); 
$p<0.001]$ and mortality rate $(5.9 \%$ vs. $24.6 \% ; p<0.001)$ were significantly higher in patients requiring invasive mechanical ventilation in comparison to non-ventilated patients $[n=328(85.6 \%)]$. With regards to in-hospital treatment, $87.1 \%$ of patients were on methylprednisolone and/or dexamethasone, $83.8 \%$ on low heparin molecular weight, $42 \%$ on azithromycin and $23 \%$ on remdesivir.

Laboratory findings are summarized in Table 1. Calprotectin (Fig. 1A), ferritin, CRP, LDH, white blood cell (WBC) count and neutrophil-to-lymphocyte ratio (NLR) were significantly higher in patients requiring invasive mechanical ventilation. No differences were found for creatinine, platelet count and D-dimer. The same findings for laboratory tests, including calprotectin (Fig. 1B), were found for the secondary endpoint (Table 1). Finally, when we analysed the biomarker levels in the ICU population $(n=100)$ for invasive mechanical ventilation, only calprotectin $[3.73 \mathrm{mg} / \mathrm{L}(2.91-8.10)$ vs. $2.79 \mathrm{mg} / \mathrm{L}(2.80-4.39)$; $p=0.001$ (Fig. 1C)], CRP [117.8 mg/L (63.3-168.8) vs. $80.1 \mathrm{mg} / \mathrm{L}(32.2-108.7) ; p=0.008]$ and ferritin [1160 ng/ $\mathrm{mL}(643-1982)$ vs. $756 \mathrm{ng} / \mathrm{mL}(510-1214) ; p=0.041]$ levels were significantly higher in patients who required invasive mechanical ventilation $[n=57(57.0 \%)]$ as compared to those who remained free of intubation $(n=43$ $(43 \%)$ ], without significant differences for the other tested biomarkers. Of note, median calprotectin levels in all analyzed subgroups of patients were over of the UNRL from a healthy population (1.63 mg/L) (Fig. 1).

Table 1 Laboratory findings on admission to ED (expressed as median [IQR])

\begin{tabular}{|c|c|c|c|c|c|c|c|}
\hline & \multirow[t]{2}{*}{ All patients } & \multicolumn{2}{|c|}{ Need for invasive mechanical ventilation } & \multirow[t]{2}{*}{$p$-value } & \multicolumn{2}{|l|}{ ICU admission } & \multirow[t]{2}{*}{$p$-value } \\
\hline & & $\begin{array}{l}\text { Non-invasive } \\
\text { mechanical venti- } \\
\text { lation }\end{array}$ & $\begin{array}{l}\text { Invasive mechanical } \\
\text { ventilation }\end{array}$ & & Non admitted & Admitted & \\
\hline $\begin{array}{l}\text { Number of patients, } \\
n(\%)\end{array}$ & 395 & $328(85.6)$ & $57(14.4)$ & - & $295(74.7)$ & $100(25.3)$ & - \\
\hline Creatinine $(\mu \mathrm{mol} / \mathrm{L})$ & $79.6(64.5-101.7)$ & $79.6(64.5-100.8)$ & $79.6(64.5-99.0)$ & 0.982 & $278.7(63.7-101.7)$ & $81.4(65.4-101.7)$ & 0.717 \\
\hline $\mathrm{LDH}(\mathrm{U} / \mathrm{L})$ & $303(240-403)$ & $293(234-387)$ & $393(311-562)$ & $<0.001$ & $1283(230-379)$ & $381(302-480)$ & $<0.001$ \\
\hline $\mathrm{CRP}(\mathrm{mg} / \mathrm{L})$ & $60.0(25.5-111.4)$ & $53.3(22.7-102.5)$ & $117.8(63.3-168.8)$ & $<0.001$ & $151.4(21.6-99.2)$ & $97.9(47.4-156.3)$ & $<0.001$ \\
\hline Ferritin $(\mu \mathrm{g} / \mathrm{L})$ & $524(257-944)$ & $470(238-803)$ & $1160(643-1982)$ & $<0.001$ & $1439(215-772)$ & $863(563-1738)$ & $<0.001$ \\
\hline WBC count $\left(* 10^{9} / \mathrm{L}\right)$ & $6.57(4.95-9.18)$ & $6.38(4.83-8.75)$ & $8.09(5.70-12.44)$ & 0.004 & $46.32(4.80-8.79)$ & $7.38(5.59-11.10)$ & 0.002 \\
\hline NLR & $4.12(2.42-7.39)$ & $4.00(2.38-6.94)$ & $5.81(3.26-9.40)$ & 0.002 & $3.81(2.29-6.37)$ & $5.45(3.12-8.93)$ & 0.001 \\
\hline $\begin{array}{l}\text { Platelet count } \\
\left(* 10^{9} / \mathrm{L}\right)\end{array}$ & $204(158-267)$ & $202(157-261)$ & $209(163-273)$ & 0.377 & $7203(156-261)$ & $205(162-274)$ & 0.574 \\
\hline D-dimer ( $\mu \mathrm{g} / \mathrm{L}$ FEU) & $612(383-1100)$ & $599(380-1064)$ & $790(506-1334)$ & 0.110 & $604(379-1130)$ & $648(421-1058)$ & 0.511 \\
\hline Calprotectin (mg/L) & $2.76(1.77-4.45)$ & $2.63(1.70-4.07)$ & $3.73(2.91-8.10)$ & $<0.001$ & $12.60(1.68-4.00)$ & $3.48(2.24-6.36)$ & $<0.001$ \\
\hline
\end{tabular}

$I Q R$ Interquartile range, $L D H$ Lactate dehydrogenase, $C R P$ C-reactive protein, WBC White blood cell, NLR Neutrophil-to-Lymphocyte Ratio, FEU Fibrinogen Equivalent Units, ICU Intensive Care Unit
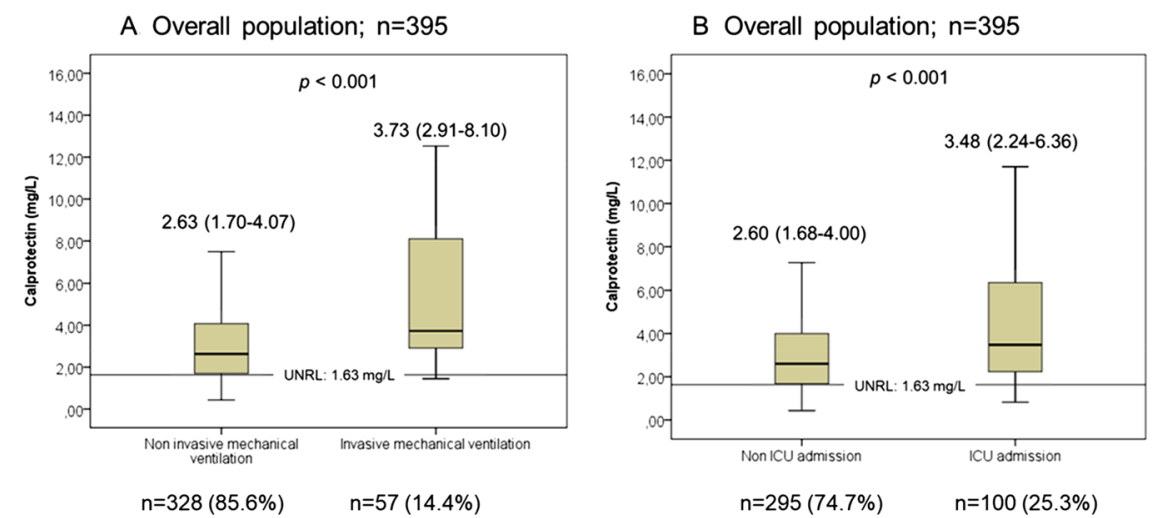

C ICU subpopulation; $n=100(25.3 \%)$

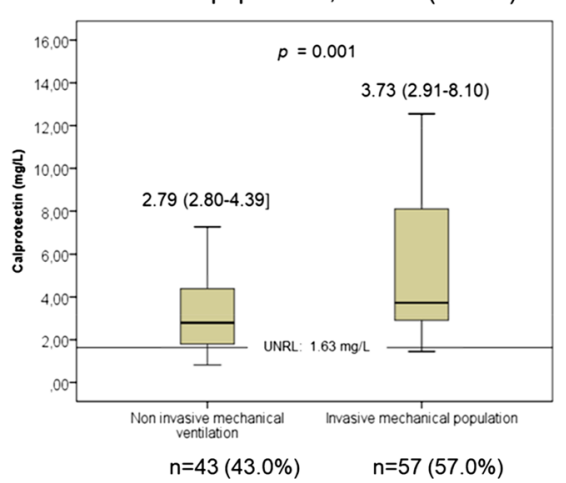

Fig. 1 Median (boxplots) values of calprotectin in patients requiring invasive mechanical ventilation (1.A) and ICU admission (1.B) in overall population and in patients requiring invasive mechanical ventilation in ICU subpopulation (1.C). In boxplots, the dark line inside the box is the median of the distribution. The bottom and the top of the boxes represent the 1 st and the 3 rd quartiles (interquartile range). UNRL: Upper normal reference limit 


\section{Patient's characteristics stratified by calprotectin levels and correlation with other biomarkers}

The median level of calprotectin in our cohort was $2.76 \mathrm{mg} / \mathrm{L}$ [IQR (1.77-4.45)]. When patients were stratified according to calprotectin quartiles, the need for invasive mechanical ventilation increased significantly from the patients in the first quartile (serum calprotectin $<1.77 \mathrm{mg} / \mathrm{L} ; n=4 / 97(4.1 \%)$ ] to the fourth quartile [serum calprotectin $\geq 4.45 \mathrm{mg} / \mathrm{L} ; n=27 / 99$ (27.3\%)] (Fig. 2). No differences between quartiles were found related to age, gender and pre-existing comorbidities. In the subgroup of patients who required invasive mechanical ventilation $(n=57)$, rate of patients with diabetes mellitus $(73.3 \%$ vs. $42.0 \% ; p=0.043)$ was significantly higher in those with elevated calprotectin levels $(\geq 3.73 \mathrm{mg} / \mathrm{L}$,

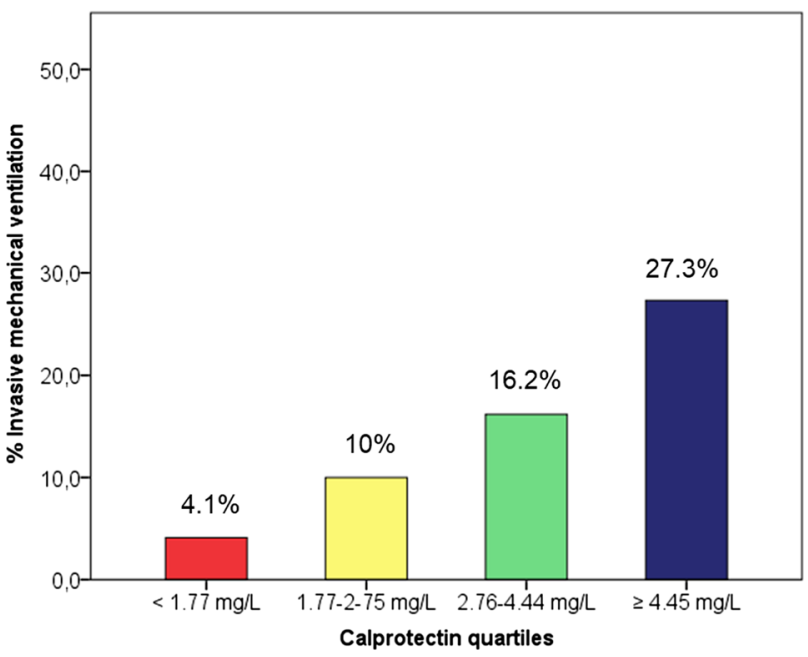

corresponding to median level) compared to ventilated patients with low calprotectin levels $(<3.73 \mathrm{mg} / \mathrm{L})$, without significant differences for other demographics and comorbidities.

Serum calprotectin levels correlated positively with levels of other biomarkers for inflammation and thrombosis, such as ferritin $(r=0.256 ; p<0.001)$, CRP $(r=0.530$; $p<0.001)$, WBC count $(r=0.534 ; p<0.001), \mathrm{LDH}$ $(r=0.492 ; \mathrm{p}<0.001), \operatorname{NLR}(\mathrm{r}=0.383 ; \mathrm{p}<0.001)$ and D-dimer $(r=0.371 ; p<0.001)$.

\section{Association of calprotectin with the severity of the disease}

In univariate analysis, clinical factors and laboratory tests that could be associated with both outcomes were analyzed. Noteworthy, no demographic variable or prior comorbidities were predictive of need for invasive mechanical ventilation and only age could predict the need for ICU admission (Supplementary Table 1). However, several laboratory tests were predictive of both endpoints (Table 2), with calprotectin yielding the numerically higher OR for invasive mechanical ventilation [OR 1.327 (95\% Confidence Interval (CI) 1.196-1.473); $p<0.001$ ] and ICU admission [OR 1.233 (95\% CI 1.127-1.349); $p<0.001]$.

In multivariate regression analysis, after adjusting for age, LDH, CRP, ferritin, WBC count and NLR, serum calprotectin has been shown as an independent predictor for need of invasive mechanical ventilation [OR 1.161 (95\% CI 1.018-1.324); $p=0.026$ ] and ICU admission [OR 1.068 (95\% CI 1.005-1.224); $p=0.034$ ] (Table 2).

Fig. 2 Rate of patients requiring invasive mechanical ventilation according to calprotectin quartiles

Table 2 Uni- and multivariate regression analysis for invasive mechanical ventilation and ICU admission

\begin{tabular}{|c|c|c|c|c|c|c|c|c|}
\hline \multirow[t]{3}{*}{ Variable } & \multicolumn{4}{|c|}{ Invasive mechanical ventilation } & \multicolumn{4}{|l|}{ ICU admission } \\
\hline & \multicolumn{2}{|l|}{ Univariate analysis } & \multicolumn{2}{|l|}{ Multivariate analysis } & \multicolumn{2}{|l|}{ Univariate analysis } & \multicolumn{2}{|l|}{ Multivariate analysis } \\
\hline & OR $(95 \% \mathrm{CI})$ & $p$-value & OR $(95 \% \mathrm{CI})$ & $p$-value & OR $(95 \% \mathrm{CI})$ & $p$-value & OR $(95 \% \mathrm{CI})$ & $p$-value \\
\hline Age & n.s & & - & - & $0.986(0.973-0.999)$ & 0.033 & $0.977(0.962-0.992)$ & 0.003 \\
\hline LDH & $1.003(1.002-1.005)$ & $<0.001$ & $1.001(1.000-1.003)$ & 0.044 & $1.003(1.002-1.004)$ & $<0.001$ & $1.002(1.000-1.004)$ & 0.014 \\
\hline CRP & $1.107(1.066-1.149)$ & $<0.001$ & $1.051(1.001-1.003)$ & 0.043 & $1.084(1.049-1.121)$ & $<0.001$ & $1.047(1.006-1.089)$ & 0.004 \\
\hline Ferritin & $1.001(1.001-1.001)$ & $<0.001$ & $1.001(1.000-1.001)$ & $<0.001$ & $1.001(1.001-1.001)$ & $<0.001$ & $1.001(1.000-1.001)$ & $<0.001$ \\
\hline WBC count & $1.121(1.044-1.203)$ & 0.012 & n.s & & $1.112(1.046-1.184)$ & $<0.001$ & $1.082(1.008-1.161)$ & 0.029 \\
\hline NLR & $1.069(1.028-1.111)$ & 0.001 & n.s & & $1.060(1.021-1.099)$ & $<0.001$ & n.s & \\
\hline Calprotectin & $1.327(1.196-1.473)$ & $<0.001$ & $1.161(1.018-1.324)$ & 0.026 & $1.233(1.127-1.349)$ & $<0.001$ & $1.068(1.005-1.224)$ & 0.034 \\
\hline
\end{tabular}

In this table, only variables with a significant OR were included

$C I$ Confidence interval, $O R$ Odd ratio, $I C U$ Intensive Care Unit, $I Q R$ Interquartile range, $L D H$ Lactate dehydrogenase, $C R P C$-reactive protein, $W B C$ White blood cell, NLR Neutrophil-to-Lymphocyte Ratio 

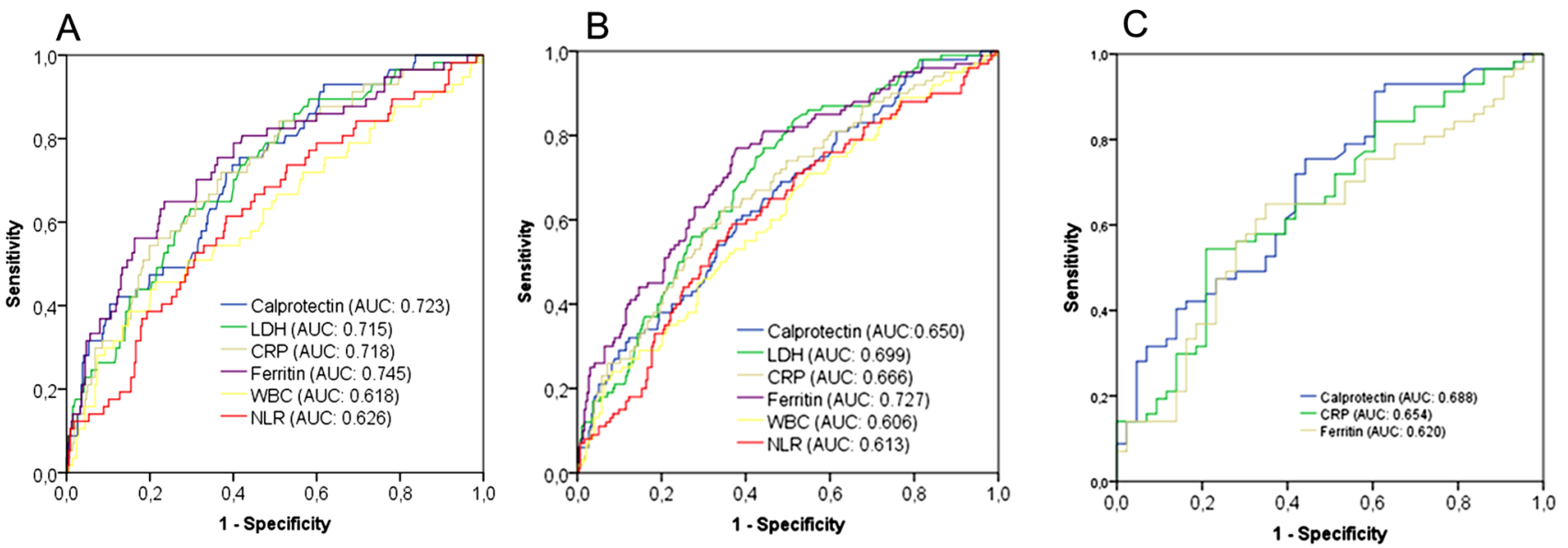

Fig. 3 Receiver operating characteristic curves of biomarker levels on arrival to ED to predict need for invasive mechanical ventilation (1.A) and ICU admission (1.B) and invasive mechanical ventilation in ICU subpopulation (1.C). 95\% CIs and p values for ROC AUCs for invasive mechanical ventilation: Calprotectin: $0.652-0.790, p<0.001$; ferritin: 0.673-0.818, $p<0.001$; CRP: 0.646-0.790, $p<0.001$; LDH: $0.645-0.786, p<0.001$; WBC count: $0.533-0.704, p=0.004$; NLR: $0.547-0.705, p=0.002 .95 \%$ CIs and $p$ values for ROC AUCs for
ICU admission: Calprotectin: 0.600-0.697, $p<0.001$; ferritin: 0.680 0.770, $p<0.001$; CRP: 0.617-0.712, $p<0.001$; LDH: 0.651-0.744, $p<0.001$; WBC count: $0.556-0.654, p=0.001$; NLR: $0.563-0.661$, $p=0.001 .95 \%$ CIs and $p$ values for ROC AUCs for invasive mechanical ventilation in ICU subpopulation: Calprotectin: 0.588-0.777, $p<0.001$; CRP: $0.553-0.747, \quad p=0.001$; ferritin: $0.517-0.715$, $p=0.036$
Table 3 Accuracy of calprotectin for predicting the need for invasive mechanical ventilation

\begin{tabular}{lllll}
\hline Cutoff $(\mathrm{mg} / \mathrm{L})$ & Sensitivity $(95 \% \mathrm{CI})$ & Specificity $(95 \% \mathrm{CI})$ & PPV (95\% CI) & NPV (95\% CI) \\
\hline $2.98^{\mathrm{a}}$ & $73.7(60.3-84.5)$ & $60.4(54.9-65.6)$ & $23.9(17.8-30.9)$ & $93.2(89.0-96.1)$ \\
$<2.23^{\mathrm{b}}$ & $86.0(74.2-93.7)$ & - & - & $94.7(89.7-96.4)$ \\
\hline
\end{tabular}

$C I$ Confidence Interval, $P P V$ Positive predictive value, $N P V$ Negative predictive value

${ }^{a}$ Optimal cut-off according to Youden index

${ }^{b}$ Optimal cut-off to rule out need for invasive mechanical ventilation

\section{Receiver operating curves for prediction of need for invasive mechanical ventilation and ICU admission}

Concerning the predictive ability for need of invasive mechanical ventilation, serum calprotectin levels showed a significant ROC AUC of 0.723 , similar to performance of ferritin (ROC AUC 0.745), CRP (ROC AUC 0.718) and LDH (ROC AUC 0.715) and significantly higher than performance of WBC count (ROC AUC 0.618) and NLR (ROC AUC 0.626) (Fig. 3A). According to Youden index, an optimal cut-off point for serum calprotectin and prediction of need for invasive mechanical ventilation, was established at $2.98 \mathrm{mg} / \mathrm{L}$, with a sensitivity of $73.7 \%$ and a specificity of $60.4 \%$ (Table 3). A second cut-off point of $2.23 \mathrm{mg} / \mathrm{L}$ maximizing the sensitivity up to $86.0 \%$ and NPV up to $94.7 \%$ was suggested to rule-out the need for invasive mechanical ventilation. Kaplan-Meier curves using a calprotectin cut-off point $<2.23 \mathrm{mg} / \mathrm{L}$ identified a significantly lower need for invasive mechanical ventilation $(5.3 \%$ vs. $20.0 \% ; p<0.001)$ (Fig. 4).

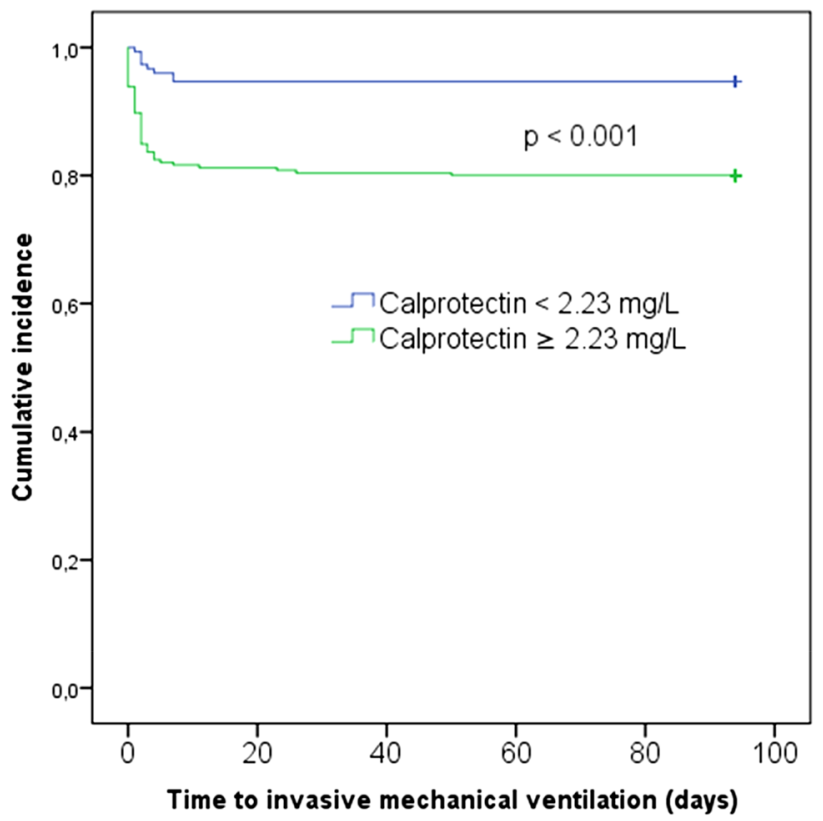

Fig. 4 Cumulative incidence of invasive mechanical ventilation stratified by serum calprotectin on admission $\leq 2.23 \mathrm{mg} / \mathrm{L}$ 
For prediction of ICU admission, serum calprotectin levels showed a significant ROC AUC of 0.650 (Fig. 3B), with an optimal cut-off point of $3.09 \mathrm{mg} / \mathrm{L}$ according to Youden index [sensitivity 60\% (95\% CI 49.7-69.7); specificity $62.4 \%$ (95\% CI 56.6-67.9)]. This accuracy was similar to those achieved by other inflammatory biomarkers, such as CRP (AUC 0.666), LDH (AUC 0.699), WBC (AUC 0.606) and NLR (AUC 0.613), but slightly lower than ferritin (AUC 0.727; p of comparison: 0.027).

Finally, we also analysed the accuracy of laboratory tests in the ICU subpopulation for predicting the need for invasive mechanical ventilation. Serum calprotectin [AUC 0.688 (95\% CI 0.588-0.777); $p<0.001]$ was the biomarker with the ROC AUC numerically higher, although without significant difference in comparison to CRP [AUC 0.654 (95\% CI 0.553-0.747); $p=0.001 ; p$ of comparison: 0.571] and ferritin [AUC 0.620 (95\% CI 0.517-0.715); $p=0.036$; $p$ of comparison 0.278] (Fig. 3C). For an optimal cut-off point of $2.81 \mathrm{mg} / \mathrm{L}$, calprotectin achieved a sensitivity of $75.4 \%$ [95\% CI 62.2-85.9] and a specificity of 55.8\% [95\% CI 39.9-70.9].

\section{Discussion}

COVID-19 is characterized by a clinical picture ranging from mild upper airway symptoms in most of patients to severe lower airway symptoms in a subgroup of patients developing acute respiratory distress syndrome that progress to respiratory failure due to intense acute lung injury [31] and cytokine storm syndrome resulting in a multiorgan failure and death [32].

Recent research confirms that neutrophils play a central role in the immunopathology of COVID-19 [33]. Thus, the characterization of neutrophil activation by the measurement of calprotectin levels in blood has emerged as a predictor of COVID-19 severity and the value of calprotectin in severe COVID-19 was recently reviewed [11, 12]. However, larger studies have been proposed to evaluate the value of calprotectin in risk stratification in severe COVID-19 [12]. The aim of our multicenter study, conducted during the second wave of pandemic in Spain was to assess the value of serum calprotectin in prediction of severe respiratory failure in hospitalized COVID-19 patients, and secondarily, of need for ICU admission. The level of serum calprotectin was measured by a fully automated turbidimetric assay [29], providing rapid test results.

The role of conventional inflammatory markers, such as NLR, CRP, LDH and ferritin, in prediction of respiratory failure has been widely reported in COVID-19 patients during the first wave of pandemic. CRP and LDH are inversely correlated to respiratory function evaluated by the partial pressure of arterial oxygen to fraction of inspired oxygen ratio $(\mathrm{PaO} 2 / \mathrm{FiO} 2)$ and are predictors of moderate-severe acute respiratory distress syndrome (ARDS) [34] with ROC AUC close to 0.8 . Herold et al. have recently reported similar results for both biomarkers, showing AUCs of 0.79 and 0.83 for CRP and LDH, respectively [35]. Although significantly higher values have been reported in patients who required mechanical ventilation, NLR was shown to be a moderate predictor of this outcome, with AUCs ranging from 0.581 [36] to 0.710 [37]. The value of ferritin for respiratory failure is also controversial. Herold et al. reported a non-significant ROC AUC of 0.72 (95\% CI 0.52-0.91; $p=0.064$ ) for need for mechanical ventilation, lower than the accuracy achieved by CRP (ROC AUC: 0.79) and LDH (ROC AUC 0.79) [35]. Feld et al. [38] have reported a ROC AUC of 0.681 for ferritin measured at hospital admission, consistent with a poor discriminating ability for prediction of intubation. Noteworthy, in our study, differences were not observed for some laboratory tests strongly associated with COVID-19 severity during the first wave, such as creatinine, D-dimer and platelet count. A similar finding has been recently described by Mollinedo-Gajate et al. [39] when critical or non-survivors were compared with patients with a moderate severity.

The value of calprotectin has been previously reported in other infectious diseases, with better diagnostic and prognostic performance than other biomarkers, such as procalcitonin $[15,17,40]$, as well as in inflammatory diseases such as rheumatoid arthritis, as a biomarker for disease activity [41, 42]. In COVID-19 patients, several studies have evaluated the potential role of calprotectin for prediction of need for mechanical ventilation [19], ICU admission [23, 24, 26], multiorgan failure [26] and death [23, 25, 26]. Main limitations in these studies are the small sample size and the inclusion of patients recruited in the first wave of pandemic. The first wave was more severe than the second [43] resulting in differences between values reported for some thromboinflammatory markers in comparison to the second wave [39].

This study, performed in a cohort of 395 patients recruited during the second wave of pandemic in four Spanish hospitals, confirms the role of calprotectin in risk stratification at an early stage in patients presented at the emergency department, with a significant accuracy for the need of invasive mechanical ventilation and ICU admission. In the study performed by Shi et al. [19], the ROC AUC of serum calprotectin, measured by an ELISA assay in samples collected during the first 2 days of hospitalization, to predict the need for mechanical ventilation was 0.794 , which is slightly higher than ROC AUC obtained in our study (0.723). This disagreement could be explained by differences in the time for collection of samples as well as differences in relation to the assay used for measurement, as recently described by Van Hoovels et al. [44]. This accuracy is similar to that 
achieved for prediction of need for mechanical ventilation in a small cohort of patients recruited during the first wave of pandemic, in which calprotectin, measured with the same assay and in the same type of sample, yielded a ROC AUC of 0.723 (95\% CI $0.563-0.883 ; p=0.032$ ), with an optimal cut-off point, according to Youden index, of $3.0 \mathrm{mg} / \mathrm{L}$ (sensitivity $78 \%$ and specificity 40\%) [25] (data not published). The cut-off point reported in above-mentioned study is very similar to the cut-off point suggested in our multicenter study $(2.98 \mathrm{mg} / \mathrm{L})$. Moreover, we found that serum calprotectin level below $2.23 \mathrm{mg} / \mathrm{L}$ is an optimal cut-off point for identification of patients at low risk of need for mechanical ventilation. The early identification of this population can be useful for improved resource allocation and optimal care of patients with severe COVID-19.

We also reported a moderate but significant performance of serum calprotectin levels for prediction of ICU admission (AUC 0.650), similar to those previously reported by Bauer et al. (AUC 0.700) [26] and Kaya et al. (AUC 0.641) [24], but lower than that reported by Chen et al. (AUC 0.875) [23], and prediction of invasive mechanical ventilation in patients requiring management in ICU (AUC 0.688).

Finally, binary logistic regression analysis confirmed the value of calprotectin as an independent predictor of need for invasive mechanical ventilation, finding not previously reported in the literature, and ICU admission, similarly to Kaya et al. [24] (OR 1.029).

This is the study with the largest sample size evaluating the role of calprotectin as an early predictor of need for invasive mechanical ventilation and ICU admission, but it has some limitations. First, a well-defined control group including healthy individuals or patients with other respiratory infections was not included in the study design. Second, only one measurement of calprotectin and other biomarkers in samples collected on admission to the emergency department was performed and the impact of serial measurements was not evaluated. Shi et al. [19] reported increasing levels of calprotectin in patients with a deteriorating clinical condition. Third, other biomarkers previously reported as predictors of need for mechanical ventilation, such as IL-6 [35], were not measured in this study. Fourth, early warning scores, such the National Early Warning Score 2, which has been reported as predictor of severe disease, was not calculated upon admission to the Emergency Department [45]. Finally, the results for calprotectin are assay- and matrixdependent [44]; therefore, the suggested cut-off points must be confirmed for each assay, because test results cannot be used inter-changeably [44, 46-48]. Besides, Van Hoovels et al. [44] have reported that the variability (CV\%) of calprotectin measured after different storage times or after different freeze-thaw cycles was higher in serum than in EDTA-plasma. Recently, Mylemans et al. [46] have also reported a greater stability of calprotectin in EDTA-plasma resulting in higher serum calprotectin levels in comparison to plasma [44]. Noteworthy, the lack of harmonization in important preanalytical factors could hamper adequate interstudy comparison. In our study, serum tubes were centrifuged within $2 \mathrm{~h}$ after blood collection [46], immediately transferred to the secondary tube, as recommended by the manufacturer, and the samples were stored according to stability results previously reported [46].

In summary, our results support the association of neutrophil activation, characterized by elevated calprotectin levels, with COVID-19 severity. Calprotectin might be used as an early, valuable predictor of need for invasive ventilator support in COVID-19 patients. In the current situation with overwhelmed ICU and limited resources in the healthcare systems, an early identification of patients on risk of severe respiratory failure, requiring critical care management, is of key importance. The assessment of calprotectin and other biomarkers, in combination with other clinical data might be helpful for fast triage, timely allocation and effective care of COVID-19 patients.

Supplementary Information The online version contains supplementary material available at https://doi.org/10.1007/s00011-021-01516-4.

Acknowledgements We want to acknowledge the INCLIVA Biobank (PT17/0015/0049; B.0000768 ISCIII) integrated in the Spanish National Biobanks Network for their collaboration and in the Valencian Biobanking Network for the collection of samples in Hospital Clínico Universitario Valencia.

Author contributions LGGR, DMG, CRR and DAO designed the study. LGGR analyzed the data and wrote the manuscript. CRR provided statistical advice. DMG, DAO and AH reviewed critically the manuscript. CRR and LAB measured calprotectin levels in serum samples. LAB, CRR, ERB, APG, CJS, ABC, ILA, MDC and WTF collected the laboratory investigations. VEF, CBJ, MHO, MDRM, LFP, JOP, MJFR, VCR, ABF, AMGM, LPA, MGM, ERPL, ABLT, MSRB and LNM contributed to the enrolment of patients and clinical data collection. All authors reviewed and approved the final manuscript. All authors have accepted responsibility for the entire content of this manuscript and approved its submission.

Funding Gentian AS, Moss, Norway, supported the study providing reagents and other materials for measurement of calprotectin. Gentian AS did not participate in the study design, collection and analysis of data.

\section{Declarations}

Conflict of interest Aleksandra Havelka is employed by Gentian AS. Other authors state no conflict of interest.

\section{References}

1. Zhu N, Zhang D, Wang W, Li X, Yang B, Song J, et al; China Novel Coronavirus Investigating and Research Team. A Novel 
Coronavirus from Patients with Pneumonia in China, 2019. N Engl J Med. 2020;382(8):727-33.

2. Berenguer J, Ryan P, Rodríguez-Baño J, Jarrín I, Carratalà J, Pachón J, et al; COVID-19@ Spain Study Group. Characteristics and predictors of death among 4035 consecutively hospitalized patients with COVID-19 in Spain. Clin Microbiol Infect. 2020;26(11):1525-36.

3. Thompson S, Bohn MK, Mancini N, Loh TP, Wang CB, Grimmler $\mathrm{M}$, et al; IFCC Taskforce on COVID-19. IFCC Interim Guidelines on Biochemical/Hematological Monitoring of COVID-19 Patients. Clin Chem Lab Med. 2020;58(12):2009-16.

4. Meizlish ML, Pine AB, Bishai JD, Goshua G, Nadelmann $\mathrm{ER}$, Simonov $\mathrm{M}$, et al. A neutrophil activation signature predicts critical illness and mortality in COVID-19. Blood Adv. 2021;5(5):1164-77.

5. García de Guadiana-Romualdo L, Calvo Nieves MD, Rodríguez Mulero MD, Calcerrada Alises I, Hernández Olivo M, Trapiello Fernández W, et al. MR-proADM as marker of endotheliitis predicts COVID-19 severity. Eur J Clin Invest. 2021;51(5):e13511.

6. Barnes BJ, Adrover JM, Baxter-Stoltzfus A, Borczuk A, Cools-Lartigue J, Crawford JM, et al. Targeting potential drivers of COVID-19: Neutrophil extracellular traps. J Exp Med. 2020;217(6):e20200652.

7. Dennison D, Al Khabori M, Al Mamari S, Aurelio A, Al Hinai H, Al Maamari K, et al. Circulating activated neutrophils in COVID19: an independent predictor for mechanical ventilation and death. Int J Infect Dis. 2021;106:155-9.

8. Rada B. Neutrophil Extracellular Traps. Methods Mol Biol. 1982;2019:517-28.

9. Yang SC, Tsai YF, Pan YL, Hwang TL. Understanding the role of neutrophils in acute respiratory distress syndrome. Biomed $\mathrm{J}$. 2020;10:S2319-4170(20)30149-9.

10. Veras FP, Pontelli MC, Silva CM, Toller-Kawahisa JE, de Lima M, Nascimento DC, et al. SARS-CoV-2-triggered neutrophil extracellular traps mediate COVID-19 pathology. J Exp Med. 2020;217(12):e20201129.

11. Udeh R, Advani S, de Guadiana Romualdo LG, Dolja-Gore X. Calprotectin, an emerging biomarker of interest in COVID-19: a systematic review and meta-analysis. J Clin Med. 2021;10(4):775.

12. Mahler M, Meroni PL, Infantino M, Buhler KA, Fritzler MJ. Circulating Calprotectin as a Biomarker of COVID-19 Severity. Expert Rev Clin Immunol. 2021:1-13.

13. Azramezani Kopi T, Shahrokh S, Mirzaei S, Asadzadeh Aghdaei $\mathrm{H}$, Amini KA. The role of serum calprotectin as a novel biomarker in inflammatory bowel diseases: a review study. Gastroenterol Hepatol Bed Bench. 2019;12(3):183-9.

14. Ometto F, Friso L, Astorri D, Botsios C, Raffeiner B, Punzi L, Doria A. Calprotectin in rheumatic diseases. Exp Biol Med (Maywood). 2017;242(8):859-73.

15. Bartáková E, Štefan M, Stráníková A, Pospíšilová L, Arientová S, Beran O, Blahutová M, Máca J, Holub M. Calprotectin and calgranulin C serum levels in bacterial sepsis. Diagn Microbiol Infect Dis. 2019;93(3):219-26.

16. Havelka A, Sejersen K, Venge P, Pauksens K, Larsson A. Calprotectin, a new biomarker for diagnosis of acute respiratory infections. Sci Rep. 2020;10(1):4208.

17. Jonsson N, Nilsen T, Gille-Johnson P, Bell M, Martling CR, Larsson A, Mårtensson J. Calprotectin as an early biomarker of bacterial infections in critically ill patients: an exploratory cohort assessment. Crit Care Resusc. 2017;19(3):205-13.

18. Dale I, Brandtzaeg P, Fagerhol MK, Scott H. Distribution of a new myelomonocytic antigen (L1) in human peripheral blood leukocytes. Immunofluorescence and immunoperoxidase staining features in comparison with lysozyme and lactoferrin. Am J Clin Pathol. 1985;84(1):24-34.
19. Shi H, Zuo Y, Yalavarthi S, Gockman K, Zuo M, Madison JA, Blair C, et al. Neutrophil calprotectin identifies severe pulmonary disease in COVID-19. J Leukoc Biol. 2021;109:67-72.

20. Silvin A, Chapuis N, Dunsmore G, Goubet AG, Dubuisson A, Derosa L, et al. Elevated calprotectin and abnormal myeloid cell subsets discriminate severe from mild COVID-19. Cell. 2020;182:1401-1418.e18.

21. Hanssen NMJ, Spaetgens B, Nagareddy PR, Murphy AJ. DAMPening mortality in COVID-19: therapeutic insights from basic cardiometabolic studies on S100A8/A9. Circulation. 2021;143(10):971-3.

22. Cherubini F, Cristiano A, Valentini A, Bernardini S, Nuccetelli M. Circulating calprotectin as a supporting inflammatory marker in discriminating SARS-CoV-2 infection: an observational study. Inflamm Res. 2021;70(6):687-94.

23. Chen L, Long X, Xu Q, Tan J, Wang G, Cao Y, et al. Elevated serum levels of S100A8/A9 and HMGB1 at hospital admission are correlated with inferior clinical outcomes in COVID-19 patients. Cell Mol Immunol. 2020;17(9):992-4.

24. Kaya T, Yaylacı S, Nalbant A, Yıldırım İ, Kocayiğit H, Çokluk E, et al. Serum calprotectin as a novel biomarker for severity of COVID-19 disease. Ir J Med Sci. 2021;27:1-6.

25. Luis García de Guadiana Romualdo, Mulero MDR, Olivo MH, Rojas CR, Arenas VR, Morales MG, et al. Circulating levels of GDF-15 and calprotectin for prediction of in-hospital mortality in COVID-19 patients: A case series. J Infect. 2021;82(2):e40-e42.

26. Bauer W, Diehl-Wiesenecker E, Ulke J, Galtung N, Havelka A, Hegel JK, et al. Outcome prediction by serum calprotectin in patients with COVID-19 in the emergency department. J Infect. 2021;82(4):84-123.

27. World Health Organization. Laboratory testing for coronavirus disease (COVID-19) in suspected human cases. https://www.who. int/publications-detail/laboratory-testing-for-2019-novel-coron avirus-insuspected-human-cases-20200117. Accessed 25 August 2020.

28. Spanish Health Ministry. Documento técnico. Manejo en urgencias del COVID-19. www.mscbs.gob.es/profesionales/saludPubli $\mathrm{ca} /$ ccayes/alertasActual/nCov/documentos.htm.

29. Nilsen T, Sunde K, Larsson A. A new turbidimetric immunoassay for serum calprotectin for fully automatized clinical analysers. J Inflamm (Lond). 2015;12:45.

30. Berlin DA, Gulick RM, Martinez FJ. Severe Covid-19. N Engl J Med. 2020;383(25):2451-60.

31. Lai CC, Shih TP, Ko WC, Tang HJ, Hsueh PR. Severe acute respiratory syndrome coronavirus 2 (SARS-CoV-2) and coronavirus disease-2019 (COVID-19): The epidemic and the challenges. Int J Antimicrob Agents. 2020;55(3):105924.

32. Ragab D, Salah Eldin H, Taeimah M, Khattab R, Salem R. The COVID-19 cytokine storm; what we know so far. Front Immunol. 2020;11:1446.

33. Tomar B, Anders HJ, Desai J, Mulay SR. Neutrophils and neutrophil extracellular traps drive necroinflammation in COVID-19. Cells. 2020;9(6):1383.

34. Poggiali E, Zaino D, Immovilli P, Rovero L, Losi G, Dacrema $\mathrm{A}$, et al. Lactate dehydrogenase and C-reactive protein as predictors of respiratory failure in CoVID-19 patients. Clin Chim Acta. 2020;509:135-8.

35. Herold T, Jurinovic V, Arnreich C, Lipworth BJ, Hellmuth JC, von Bergwelt-Baildon M, et al. Elevated levels of IL- 6 and CRP predict the need for mechanical ventilation in COVID-19. J Allergy Clin Immunol. 2020;146(1):128-136.e4.

36. Ramos-Peñafiel CO, Santos-González B, Flores-López EN, Galván-Flores F, Hernández-Vázquez L, Santoyo-Sánchez A, et al. Usefulness of the neutrophil-to-lymphocyte, monocyte-to-lymphocyte and lymphocyte-to-platelet ratios for the 
prognosis of COVID-19-associated complications. Gac Med Mex. 2020;156(5):405-11.

37. Nair PR, Maitra S, Ray BR, Anand RK, Baidya DK, Subramaniam R. Neutrophil-to-lymphocyte ratio and platelet-to-lymphocyte ratio as predictors of the early requirement of mechanical ventilation in COVID-19 patients. Indian J Crit Care Med. 2020;24(11):1143-4.

38. Feld J, Tremblay D, Thibaud S, Kessler A, Naymagon L. Ferritin levels in patients with COVID-19: a poor predictor of mortality and hemophagocytic lymphohistiocytosis. Int J Lab Hematol. 2020;42(6):773-9.

39. Mollinedo-Gajate I, Villar-Álvarez F, Zambrano-Chacón MLÁ, Núñez-García L, de la Dueña-Muñoz L, López-Chang C, et al. First and Second Waves of Coronavirus Disease 2019 in Madrid, Spain: Clinical Characteristics and Hematological Risk Factors Associated With Critical/Fatal Illness. Crit Care Explor. 2021;3(2):e0346.

40. Larsson A, Tydén J, Johansson J, Lipcsey M, Bergquist M, Kultima K, et al. Calprotectin is superior to procalcitonin as a sepsis marker and predictor of 30-day mortality in intensive care patients. Scand J Clin Lab Invest. 2020;80(2):156-61.

41. Hurnakova J, Hulejova H, Zavada J, Komarc M, Cerezo LA, Mann $\mathrm{H}$, et al. Serum calprotectin may reflect inflammatory activity in patients with active rheumatoid arthritis despite normal to low C-reactive protein. Clin Rheumatol. 2018;37(8):2055-62.

42. Jarlborg M, Courvoisier DS, Lamacchia C, Martinez Prat L, Mahler M, Bentow C, et al; physicians of the Swiss Clinical Quality Management (SCQM) registry. Serum calprotectin: a promising biomarker in rheumatoid arthritis and axial spondyloarthritis. Arthritis Res Ther. 2020;22(1):105.

43. Iftimie S, López-Azcona AF, Vallverdú I, Hernández-Flix S, de Febrer G, Parra S, et al. First and second waves of coronavirus disease-19: A comparative study in hospitalized patients in Reus, Spain. PLoS One. 2021;16:e0248029.

44. Van Hoovels L, Vander Cruyssen B, Bogaert L, Van den Bremt S, Bossuyt X. Pre-analytical and analytical confounders of serum calprotectin as a biomarker in rheumatoid arthritis. Clin Chem Lab Med. 2019;58(1):40-9.

45. Myrstad M, Ihle-Hansen H, Tveita AA, Andersen EL, Nygård $S$, Tveit A, et al. National early warning score 2 (NEWS2) on admission predicts severe disease and in-hospital mortality from Covid-19 - a prospective cohort study. Scand J Trauma Resusc Emerg Med. 2020;28(1):66.

46. Mylemans M, Nevejan L, Van Den Bremt S, Stubbe M, Cruyssen BV, Moulakakis C, et al. Circulating calprotectin as biomarker in neutrophil-related inflammation: pre-analytical recommendations and reference values according to sample type. Clin Chim Acta. 2021;517:149-215.

47. Gao J, Ulvik A, McCann A, Ueland PM, Meyer K. Microheterogeneity and preanalytical stability of protein biomarkers of inflammation and renal function. Talanta. 2021;223(Pt 1):121774.

48. Nordal HH, Fagerhol MK, Halse AK, Hammer HB. Calprotectin (S100A8/A9) should preferably be measured in EDTA-plasma; results from a longitudinal study of patients with rheumatoid arthritis. Scand J Clin Lab Invest. 2018;78(1-2):102-8.

Publisher's Note Springer Nature remains neutral with regard to jurisdictional claims in published maps and institutional affiliations. 


\section{Authors and Affiliations}

Luis García de Guadiana-Romualdo ${ }^{1}$ (1) - Carlos Rodríguez Rojas² • Daniel Morell-García ${ }^{3,4}$ • David Andaluz-Ojeda ${ }^{5}$. María Dolores Rodríguez Mulero ${ }^{6}$. Enrique Rodríguez-Borja ${ }^{2} \cdot$ Antonieta Ballesteros-Vizoso $^{3}$ - María Dolores Calvo ${ }^{7}$. Lourdes Albert-Botella ${ }^{8}$. Adela Pozo Giráldez ${ }^{2}$. Isabel Llompart-Alabern ${ }^{3}$. Cristina Bolado Jiménez ${ }^{9}$. Marta Hernández Olivo ${ }^{10}$. Lucía Fernández Presa ${ }^{11}$. Juan Ortega-Pérez ${ }^{4,12}$. María José Fora Romero ${ }^{9}$. Valerio Campos-Rodríguez ${ }^{13}$. Andrea Ballester Férriz ${ }^{11}$. Alexandra M. Guiu-Martí ${ }^{12}$. Leyre Pinilla Arribas ${ }^{9}$. María Galindo Martínez ${ }^{6}$ Edwin R. Puerto-Lara ${ }^{12}$. Ana Belen López Tarazaga ${ }^{9}$. María Salomé Ros Braquehais ${ }^{10}$. Leonor Nogales Martín ${ }^{5}$. Celia Juez Santamaría ${ }^{14}$. Wysalli Trapiello Fernández ${ }^{9}$. Virginia Espinilla Fernández ${ }^{15}$. Aleksandra Havelka $^{16,17}$. María Dolores Albaladejo-Otón ${ }^{1}$

Aleksandra Havelka

aleksandra.havelka@gentian.com

1 Laboratory Medicine Department, Hospital Universitario Santa Lucía, Paraje Los Arcos, Calle Mezquita s/n, CP 30202 Cartagena, Spain

2 Laboratory of Biochemistry, Hospital Clínico Universitario Valencia, Valencia, Spain

3 Laboratory Medicine Department, Son Espases University Hospital, Palma de Mallorca, Spain

4 Health Research Institute of Balearic Islands, Son Espases University Hospital, Palma de Mallorca, Spain

5 Intensive Care Medicine Department, Hospital Clínico Universitario, Valladolid, Spain

6 Critical Care Unit, Hospital Universitario Santa Lucía, Cartagena, Spain

7 Laboratory Medicine Department, Hospital Clínico Universitario, Valladolid, Spain

8 Laboratory Medicine Department. Hospital Ibermutuamur, Murcia, Spain
9 Emergency Department, Hospital Clínico Universitario, Valladolid, Spain

10 Pneumology Department, Hospital Universitario Santa Lucía, Cartagena, Spain

11 Pneumology Department, Hospital Clínico Universitario Valencia, Valencia, Spain

12 Emergency Medicine Department, Son Espases University Hospital, Palma de Mallorca, Spain

13 Internal Medicine Department, Hospital Universitario Santa Lucía, Cartagena, Spain

14 Department of Hospital Pharmacy, Hospital Universitario Santa Lucía, Cartagena, Spain

15 Preventive Medicine Department, Hospital Clínico Universitario, Valladolid, Spain

16 Department of Molecular Medicine and Surgery, Karolinska Institute, Stockholm, Sweden

17 Gentian AS, Moss, Norway 